\title{
INVERSE TRANSFORMS OF PRODUCTS OF LEGENDRE TRANSFORMS
}

\author{
R. V. CHURCHILL AND C. L. DOLPH
}

1. Introduction. The finite linear integral transformation

$$
T\{F(x)\}=\int_{-1}^{1} F(x) P_{n}(x) d x=f(n) \quad(n=0,1,2, \cdots)
$$

of functions $F(x)$ defined on the interval $-1 \leqq x \leqq 1$, where $P_{n}(x)$ denotes the Legendre polynomials, serves as the basis of an operational calculus. The sequence of numbers $f(n)$ is called the Legendre transform of the function $F(x)$.

The fundamental operational property of this transformation

$$
T\left\{\frac{d}{d x}\left[\left(1-x^{2}\right) \frac{d F}{d x}\right]\right\}=-n(n+1) f(n)
$$

is obtained by applying successive integration by parts to the integral represented by the left-hand member of this equation so that the new integrand is the product of $F$ by $\left[\left(1-x^{2}\right) P_{n}^{\prime}\right]^{\prime}$, and by replacing this second factor by $-n(n+1) P_{n}$ according to Legendre's differential equation [1]. Thus certain boundary value problems in ordinary and partial differential equations that involve the differential form appearing in the braces of equation (2) reduce to problems containing one less independent variable when written in terms of the transform of the unknown function. This has been illustrated in the literature $[1 ; 2]$. The role played by boundary conditions and differential forms in determining appropriate integral transforms for the reduction of given types of boundary value problems was indicated in an earlier paper [3].

The inverse $T^{-1}\{f(n)\}$ denotes a function $F(x)$ whose transform is $f(n)$. If $F(x)$ satisfies conditions under which it is represented by its Legendre series [4], then it follows from the formulas for the coefficients in that series that

$$
F(x)=\sum_{n=0}^{\infty}\left(n+\frac{1}{2}\right) f(n) P_{n}(x)=T^{-1}\{f(n)\} \quad(-1<x<1) .
$$

The operational calculus based on Legendre transforms has been developed little beyond the stage of using formulas (1)-(3) above. 1953.

Presented to the Society, September 3, 1952; received by the editors February 16, 
The operator $T$ replaces a differential form in $F$ by an algebraic operation on $f$, according to formula (2), and if the problem in $f$ can be solved then $F(x)$ is given, at least formally, by equation (3). An examination of these operations shows that they represent a systematic way of replacing known and unknown functions in a boundary value problem by their Legendre series and then solving for the unknown coefficients. Then the expansion (3) gives the unknown function. The operational calculus can be extended beyond this primitive stage by deriving a more complete set of properties of the operator $T$.

A convolution property of the transformation is one that expresses the inverse transform of the product of two transforms in terms of the two object functions without direct recourse to the basic inversion formula (3). As in the operational calculus based upon Fourier and Laplace transforms, the convolution property makes possible a substantial extension of tables of transforms and it leads to alternate forms, even closed forms, of solutions of many boundary value problems. This property, which is necessary for the full development of the operational calculus, will be derived here.

2. The convolution property. Let $F(x)$ and $G(x)$ denote continuous functions on the interval $-1 \leqq x \leqq 1$. If $f(n)$ and $g(n)$ represent their Legendre transforms, then

$$
\begin{aligned}
f(n) g(n)= & \int_{0}^{\pi} F(\cos \mu) P_{n}(\cos \mu) \sin \mu d \mu \\
& \cdot \int_{0}^{\pi} G(\cos \lambda) P_{n}(\cos \lambda) \sin \lambda d \lambda \\
= & \int_{0}^{\pi} F(\cos \mu) \sin \mu\left[\int_{0}^{\pi} G(\cos \lambda) P_{n}(\cos \lambda)\right. \\
& \left.\cdot P_{n}(\cos \mu) \sin \lambda d \lambda\right] d \mu .
\end{aligned}
$$

With the aid of the addition formula [5]

$$
P_{n}(\cos \lambda) P_{n}(\cos \mu)=\frac{1}{\pi} \int_{0}^{\pi} P_{n}(\cos \nu) d \alpha
$$

where

$$
\cos \nu=\cos \lambda \cos \mu+\sin \lambda \sin \mu \cos \alpha,
$$

the product can be written in the form 
(6)

$$
\begin{aligned}
f(n) g(n)= & \frac{1}{\pi} \int_{0}^{\pi} F(\cos \mu) \sin \mu \\
& \cdot\left[\int_{0}^{\pi} \int_{0}^{\pi} G(\cos \lambda) P_{n}(\cos \nu) \sin \lambda d \alpha d \lambda\right] d \mu .
\end{aligned}
$$

The iterated integral inside the brackets can be interpreted as a surface integral over a unit hemisphere $X^{2}+Y^{2}+Z^{2}=1, Z \geqq 0$, as follows. Let the fixed value of $\mu(0<\mu<\pi)$ represent arc length on the semicircle $X^{2}+Y^{2}=1, Z=0, Y>0$, measured from the point $A(1,0,0)$, and let $B$ denote the terminal point of the arc (Fig. 1). If

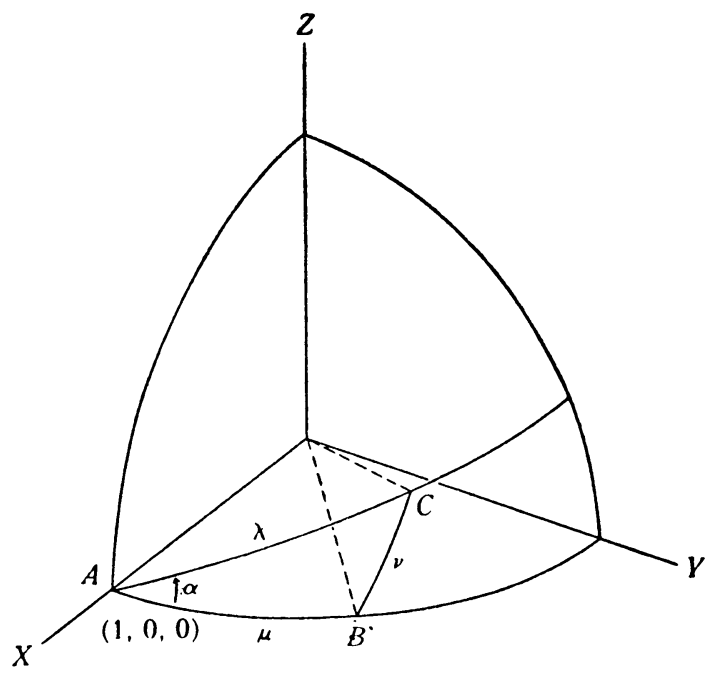

FigURE 1

$C$ is any point on the hemisphere, let $\lambda$ represent the arc $A C$ of the great circle through $A$ and $C$, and let $\alpha$ denote the angle at $A$. Thus $\alpha$ and $\lambda$ are coordinates of the point $C$. Moreover, sin $\lambda d \alpha d \lambda$ represents an element of area of the surface so that the integral is the surface integral of the function $G(\cos \lambda) P_{n}(\cos \nu)$, where $\cos \nu$ is given by equation (5) as a function of those coordinates. In fact, according to the cosine law for the spherical triangle $A B C$, equation (5) shows that $\nu$ represents the side $B C$ of the triangle, where $B C$ is the arc of a great circle.

If $\beta$ represents the angle at $B$, then $\beta$ and $\nu$, both ranging from 0 to $\pi$, represent new coordinates of the point $C$ and the area element is $\sin \nu d \beta d \nu$. Also,

$$
\cos \lambda=\cos \mu \cos \nu+\sin \mu \sin \nu \cos \beta,
$$


so that the integral inside the brackets in equation (6) can be written

(8) $\int_{0}^{\pi} \int_{0}^{\pi} G(\cos \mu \cos \nu+\sin \mu \sin \nu \cos \beta) P_{n}(\cos \nu) \sin \nu d \beta d \nu$,

a result that also follows with the aid of the Jacobian of the coordinate transformation.

Upon substituting the integral (8) into equation (6) and changing the order of integration, we obtain the formula

$$
f(n) g(n)=\int_{0}^{\pi} P_{n}(\cos \nu) \sin \nu
$$

$$
\left[\frac{1}{\pi} \int_{0}^{\pi} \int_{0}^{\pi} F(\cos \mu) G(\cos \lambda) \sin \mu d \mu d \beta\right] d \nu,
$$

where $\cos \lambda$ is given by equation (7). The expression in the brackets is a function $H(\cos \nu)$ whose Legendre transform is the product $f(n) g(n)$. In this sense $H(x)$ is the convolution $F(x) * G(x)$ of the functions $F(x)$ and $G(x)$.

Our expression for $H(x)$, as exhibited in equation (9),

$$
\begin{aligned}
H(\cos \nu)= & \frac{1}{\pi} \int_{0}^{\pi} F(\cos \mu) \sin \mu d \mu \\
& \cdot \int_{0}^{\pi} G(\cos \mu \cos \nu+\sin \mu \sin \nu \cos \beta) d \beta,
\end{aligned}
$$

can be thrown into a form having geometrical significance in connection with Fig. 1 if we replace the variables $\mu, \nu$, and $\beta$ by $\lambda, \mu$, and $\alpha$, respectively. Our convolution then becomes

$$
\begin{aligned}
H(\cos \mu)= & \frac{1}{\pi} \int_{0}^{\pi} F(\cos \lambda) \sin \lambda d \lambda \\
& \cdot \int_{0}^{\pi} G(\cos \lambda \cos \mu+\sin \lambda \sin \mu \cos \alpha) d \alpha .
\end{aligned}
$$

In view of Fig. 1 and the cosine law (5), the argument of $G$ here is $\cos \nu ; \sin \lambda d \lambda d \alpha$ represents the area element $d S$ of the upper hemispherical surface $S$ in terms of the coordinates $\alpha$ and $\lambda$, so that

$$
H(\cos \mu)=F(\cos \mu) * G(\cos \mu)=\frac{1}{\pi} \iint_{S} F(\cos \lambda) G(\cos \nu) d S
$$

that is, our convolution is twice the average value of $F(\cos \lambda) G(\cos \nu)$ over the hemisphere $S$. 
According to equation (9) the convolution (10) or (11) is the operation on $F(x)$ and $G(x)$ that corresponds to the multiplication of $f(n)$ by $g(n)$ :

$$
f(n) g(n)=T\{H(x)\}=T\{F(x) * G(x), .
$$

There is an interesting resemblance between our convolution (11) and the convolution corresponding to finite Fourier transforms [6]. The one-dimensional analogue of the integral in equation (11) with $\alpha=0$ in Fig. 1 and with $S$ replaced by the unit circle $A B A$, so that $\nu=\lambda-\mu$, is essentially the convolution for finite Fourier transforms.

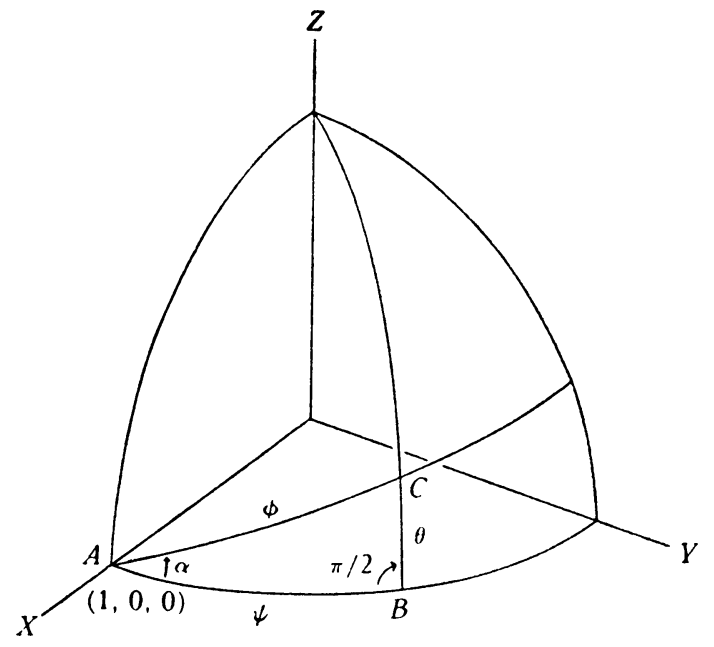

FiguRe 2

3. Other expressions for the convolution. In the interated integrals of equation (10) let us introduce the new variables

$$
\psi=\frac{\pi}{2}-\alpha, \quad \theta=\frac{\pi}{2}-\lambda ;
$$

then

$$
\begin{aligned}
H(\cos \mu)= & \frac{1}{\pi} \int_{-\pi / 2}^{\pi / 2} F(\sin \theta) \cos \theta d \theta \\
& \cdot \int_{-\pi / 2}^{\pi / 2} G(\sin \theta \cos \mu+\cos \theta \sin \mu \sin \psi) d \psi .
\end{aligned}
$$

If we interpret $\psi$ and $\theta$ as the rectangular coordinates of points $C$ on the unit spherical surface as indicated in Fig. 2, then $\cos \theta d \psi d \theta$ 
represents an element of area on the surface. The region of integration of the surface integral is the front hemispherical surface $X \geqq 0$. Equation (13) reduces to a good alternate formula for the convolution when its integral is written in terms of the new coordinates $\alpha$ and $\phi$ of points $C$ (Fig. 2).

The front hemisphere is covered by points $C$ when $\alpha$ and $\phi$ vary from 0 to $2 \pi$ and 0 to $\pi / 2$, respectively. The area element is represented by $\sin \phi d \alpha d \phi$. In the right spherical triangle $A B C$ of Fig. 2, $\sin \theta=\sin \alpha \sin \phi, \quad \tan \psi=\cos \alpha \tan \phi, \quad \cos \phi=\cos \theta \cos \psi$ and the argument of the function $G$ in equation (13) becomes $\sin \theta \cos \mu+\cos \theta \cos \psi \tan \psi \sin \mu$

$$
=\sin \phi(\sin \alpha \cos \mu+\cos \alpha \sin \mu)=\sin \phi \sin (\alpha+\mu) .
$$

According to equation (13) then the formula for our convolution $F(\cos \mu) * G(\cos \mu)$ can be written

$$
\begin{gathered}
H(\cos \mu)=\frac{1}{\pi} \int_{0}^{\pi / 2} \sin \phi d \phi \int_{0}^{2 \pi} F(\sin \phi \sin \alpha) \\
\cdot G[\sin \phi \sin (\alpha+\mu)] d \alpha .
\end{gathered}
$$

The more symmetric form

$$
\begin{aligned}
H(\cos \mu)= & \frac{1}{\pi} \int_{0}^{\pi / 2} \sin \phi d \phi \int_{0}^{2 \pi} F\left[\sin \phi \sin \left(\beta-\frac{\mu}{2}\right)\right] \\
& \cdot G\left[\sin \phi \sin \left(\beta+\frac{\mu}{2}\right)\right] d \beta
\end{aligned}
$$

is obtained by making the substitution $\alpha=\beta-\mu / 2$ in the second integral of equation (14) and noting that the integrand is periodic in $\beta$ with period $2 \pi$. Also, a simple substitution enables us to write $\cos \alpha$ and $\cos (\alpha+\mu)$ in place of $\sin \alpha$ and $\sin (\alpha+\mu)$, respectively, in equation (14).

In equation (15) let the substitutions

$$
x=\cos \mu, \quad y=\sin \phi \sin \left(\beta-\frac{\mu}{2}\right), \quad z=\sin \phi \sin \left(\beta+\frac{\mu}{2}\right)
$$

be made. We find that

and that

$$
\frac{\partial(y, z)}{\partial(\beta, \phi)}=\sin \phi \cos \phi \sin \mu
$$




$$
\frac{(z+y)^{2}}{1+x}+\frac{(z-y)^{2}}{1-x}=2 \sin ^{2} \phi .
$$

It follows readily that equation (15) takes the form

$$
H(x)=\frac{1}{\pi} \iint_{E(x)} F(y) G(z)\left(1-x^{2}-y^{2}-z^{2}+2 x y z\right)^{-1 / 2} d y d z,
$$

where $E(x)$ is the interior of the ellipse

$$
y^{2}+z^{2}-2 x y z=1-x^{2}
$$

for each fixed $x(-1<x<1)$.

4. Remarks. We have assumed above that the functions $F(x)$ and $G(x)$ are continuous. Let this condition be relaxed to the extent that those functions are assumed to be bounded and Riemann integrable on the interval $-1 \leqq x \leqq 1$. This condition is sufficient to insure the existence of the simple and double integrals used here, the identity of the double integrals with the iterated integrals, and the validity of the transformations of the double integrals, in view of known properties of such integrals [7]. Our results can therefore be stated as follows:

Theorem. Let $F(x)$ and $G(x)$ be bounded integrable functions on the interval $-1 \leqq x \leqq 1$. Then the product $f(n) g(n)$ of their Legendre transforms is the transform of the function $H(x)$ described by any one of the formulas (10), (11), (14), (15), or (16).

The authors first derived the convolution in the forms (16) and (15) by applying the inversion formula (3) to the transform $f(n) g(n)$ and then using a recent result of J. P. Vinti [8] to sum the series [9]. Then Vinti and W. C. Taylor proposed a method involving spherical triangles to arrive at formula (9). The procedure used in $\$ 2$ grew from their suggestion.

The authors are indebted to J. P. Vinti and W. C. Taylor for their contribution to this paper. They also acknowledge the assistance of G. E. Hay in simplifying one of the formulas. Others of their colleagues contributed helpful suggestions.

\section{REFERENCES}

1. C. J. Tranter, Integral transforms in mathematical physics, Methuen Monograph, Wiley, 1951, pp. 96-100. 1-8.

2. —_, Legendre transforms, Quart. J. Math. Oxford Ser. (2) vol. 1 (1950) pp. 
3. R. V. Churchill, Integral transforms and boundary value problems, Amer. Math. Monthly vol. 59 (1952) pp. 149-155.

4. E. W. Hobson, Theory of spherical and ellipsoidal harmonics, Cambridge University Press, 1931, p. 329.

5. T. M. MacRobert, Spherical harmonics, Dutton, 1927, p. 138.

6. R. V. Churchill, Modern operational mathematics, McGraw-Hill, 1944, p. 274.

7. E. W. Hobson, Theory of functions of a real variable, Cambridge University Press, 1927, pp. 473-537.

8. J. P. Vinti, Note on a series of products of three Legendre polynomials, Proc. Amer. Math. Soc. vol. 2 (1951) pp. 19-23.

9. R. V. Churchill and C. L. Dolph, Inverse transforms of products of Legendre integral transforms, Bull. Amer. Math. Soc. Abstract 58-6-610.

UNIVERSITY OF MichigaN 